\title{
Trans-chalcone activity against Trichophyton rubrum relies on an interplay between signaling pathways related to cell wall integrity and fatty acid metabolism
}

Tamires Aparecida Bitencourt 1,2, Claudia Macedo², Matheus Eloy Franco ${ }^{1,4}$, Marina Campos Rocha 3 , Igor Sawasaki Moreli ${ }^{1}$, Bruna Aline Micheloto Cantelli ${ }^{1}$, Pablo Rodrigo Sanches ${ }^{2}$, Rene Oliveira Beleboni ${ }^{1}$, Iran Malavazi ${ }^{3}$, Geraldo Aleixo Passos ${ }^{2}$, Mozart Marins ${ }^{1}$ and Ana Lúcia Fachin ${ }^{1 *}$

\begin{abstract}
Background: Trichophyton rubrum is the main etiological agent of skin and nail infections worldwide. Because of its keratinolytic activity and anthropophilic nature, infection models based on the addition of protein substrates have been employed to assess transcriptional profiles and to elucidate aspects related to host-pathogen interactions. Chalcones are widespread compounds with pronounced activity against dermatophytes. The toxicity of transchalcone towards T. rubrum is not fully understood but seems to rely on diverse cellular targets. Within this context, a better understanding of the mode of action of trans-chalcone may help identify new strategies of antifungal therapy and reveal new chemotherapeutic targets. This work aimed to assess the transcriptional profile of T. rubrum grown on different protein sources (keratin or elastin) to mimic natural infection sites and exposed to transchalcone in order to elucidate the mechanisms underlying the antifungal activity of trans-chalcone.

Results: Overall, the use of different protein sources caused only slight differences in the transcriptional profile of $T$. rubrum. The main differences were the modulation of proteases and lipases in gene categories when T. rubrum was grown on keratin and elastin, respectively. In addition, some genes encoding heat shock proteins were upregulated during the growth of T. rubrum on keratin. The transcriptional profile of T. rubrum exposed to transchalcone included four main categories: fatty acid and lipid metabolism, overall stress response, cell wall integrity pathway, and alternative energy metabolism. Consistently, T. rubrum Mapk was strongly activated during the first hours of trans-chalcone exposure. Noteworthy, trans-chalcone inhibited genes involved in keratin degradation. The results also showed effects of trans-chalcone on fatty acid synthesis and metabolic pathways involved in acetyl-CoA supply.

Conclusion: Our results suggest that the mode of action of trans-chalcone is related to pronounced changes in fungal metabolism, including an imbalance between fatty acid synthesis and degradation that interferes with cell membrane and cell wall integrity. In addition, this compound exerts activity against important virulence factors. Taken together, trans-chalcone acts on targets related to dermatophyte physiology and the infection process.
\end{abstract}

Keywords: Chalcone, CWI, Elastin, Keratin, Dermatophyte, Transcriptional profile

\footnotetext{
* Correspondence: afachin@unaerp.br

${ }^{1}$ Unidade de Biotecnologia, Universidade de Ribeirão Preto, Av: Costábile

Romano 2201, Ribeirão Preto, SP 14096-900, Brazil

Full list of author information is available at the end of the article
}

(c) The Author(s). 2019 Open Access This article is distributed under the terms of the Creative Commons Attribution 4.0 International License (http://creativecommons.org/licenses/by/4.0/), which permits unrestricted use, distribution, and reproduction in any medium, provided you give appropriate credit to the original author(s) and the source, provide a link to the Creative Commons license, and indicate if changes were made. The Creative Commons Public Domain Dedication waiver (http://creativecommons.org/publicdomain/zero/1.0/) applies to the data made available in this article, unless otherwise stated. 


\section{Background}

Dermatophytes are a group of filamentous fungi that cause cutaneous infections in humans (anthropophilic) and animals (zoophilic). Trichophyton rubrum is the most common etiological agent of clinical cases of human dermatophytoses worldwide [1]. The infection generally involves the skin and is restricted to the cornified layers such as nails, stratum corneum, and hair. Although not lethal, dermatophytoses can compromise the quality of life of the affected individual [2].

Because of their keratinolytic and keratinophilic activity, a myriad of endo- and exoproteases have been proposed as the major virulence factors of dermatophytes. Within this context, acid and alkaline proteases are fundamental for nutrient uptake from the insoluble cornified substrates. These proteases are regulated by the simultaneous co-expression of $\mathrm{pH}$ signaling genes and regulatory heat shock proteins [3]. Seven dermatophytes genomes have been sequenced $[4,5]$, which will provide the basis for a better understanding of their pathophysiological mechanisms. Additionally, in vitro and ex vivo models that mimic host-fungal interactions have been employed in order to identify new molecular targets [6].

There is current interest in identifying new molecular targets for antifungal development since most commercially available compounds target the ergosterol biosynthetic pathway and/or cell membrane [7]. In this respect, attention has been drawn to chalcones because of their multiple fungal targets such as enzymes involved in cell wall synthesis concomitant with the inhibition of fatty acid synthesis and reduction of ergosterol content [8]. A co-culture assay of T. rubrum conidia with keratinocytes exposed to trans-chalcone demonstrated the down-regulation of known virulence factors and genes of the ergosterol pathway [9]. For these reasons, chalcones are attractive molecules with multiple fungal targets. The understanding of the mode of action of chalcones may help identify new strategies of antifungal therapy and these compounds could be used as a pharmacological probe to investigate promising fungal targets.

This study assessed the transcriptional profile of $T$. rubrum during growth on different protein sources (keratin- or elastin) that mimic the host milieu in order to elucidate the mechanisms involved in the activity of trans-chalcone against this dermatophyte. Our results indicated that trans-chalcone inhibits important virulence factors such as proteases and lipases and causes impairment in essential metabolic pathways and consequent disturbance of cell wall integrity.

\section{Results}

Data analysis

The gene expression pattern of T. rubrum mycelia grown on protein substrates and exposed to trans-chalcone was assessed using high-density oligonucleotide microarray slides that cover about 6091 genes [10], corresponding to about $70 \%$ of coding genes in the $T$. rubrum genome (http://fungi.ensembl.org/info/website/ftp/index.html). A total of 290 genes were modulated on keratin medium compared to minimal medium (control) and 62 genes were modulated on elastin medium compared to control. Noteworthy, a fewer genes modulated in elastin condition in comparison to keratin (Fig. 1).

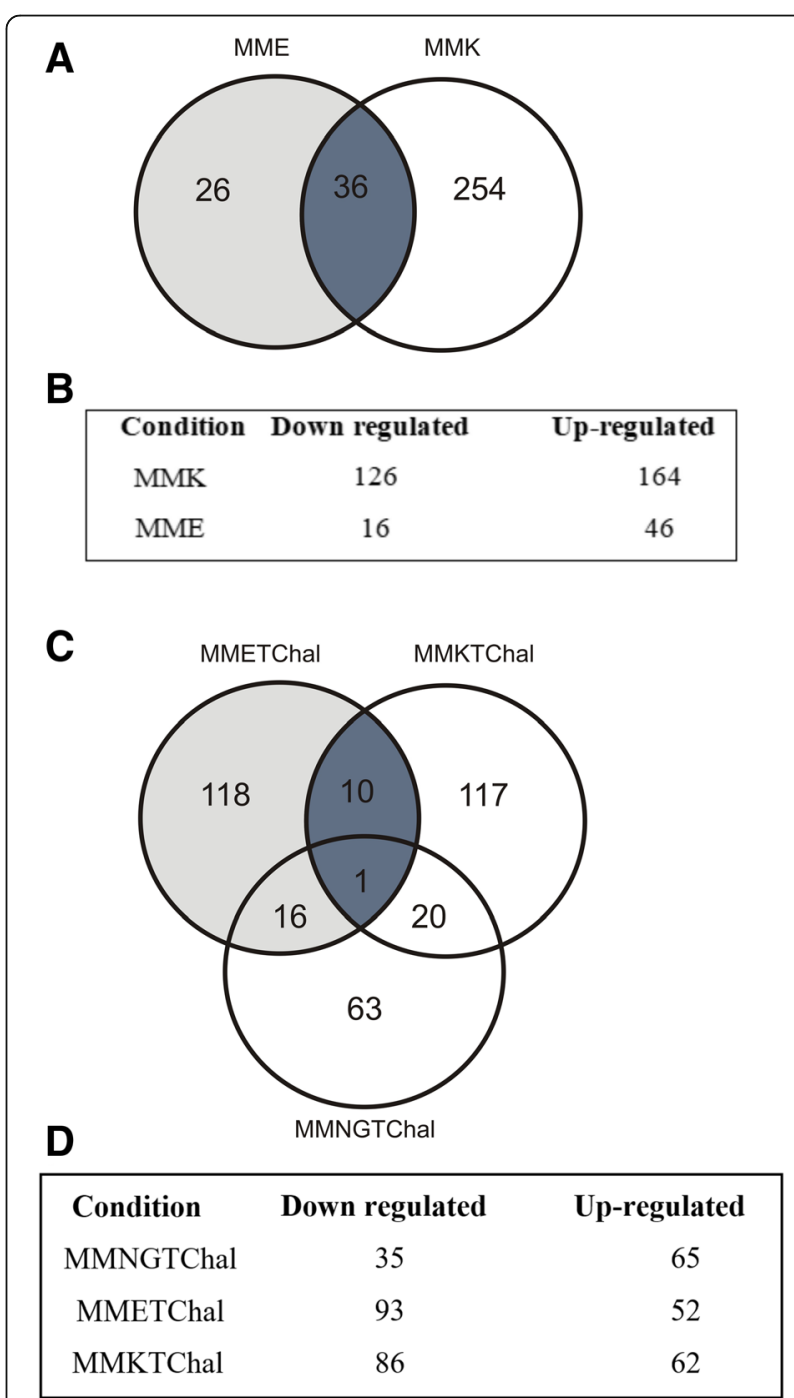

Fig. 1 Distribution of gene modulation among the conditions analyzed. (a)Venn diagram illustrating the modulation of genes during the growth of Trichophyton rubrum on elastin (MME) and keratin (MMK) compared to control (MMNG). (b) Box illustration of down- and up-regulated genes comparing the protein sources with MMNG. (c) Venn diagram illustrating the modulation of genes after exposure to trans-chalcone during growth on protein (MME + TChal and MMK + TChal) or glucose and nitrate (MMNG+TChal) compared to control (same conditions without the drug). (d) Box illustration of down- and up-regulated genes comparing the trans-chalcone conditions with no drug conditions 
After trans-chalcone exposure there were 393 genes modulated, in which 145, 148 and 100 genes modulated on minimal medium supplemented with trans-chalcone in the presence of elastin or keratin or minimal medium with glucose and nitrogen, respectively. In general, few genes were shared between conditions. Despite of MMETChal and MMKTChal showed a close number of modulated genes, changes in transcript levels were quite different, in which higher levels were shown for MMKTChal in comparison to MMETChal (Fig. 1, Additional file 1: Table S1).
Functional categorization of $T$. rubrum genes involved in the interaction with keratin and elastin substrates

The functional categorization of differentially expressed genes was performed by gene ontology (GO) using Blast2GO [11]. During T. rubrum growth on protein sources, the main categories modulated were related to signal transduction, fatty acid and lipid metabolism, proteolysis, regulation of transcription, transport, metabolic processes, and an elevated number of hypothetical proteins with unknown functions (Fig. 2). Overall, growth on different protein sources caused only slight differences in the gene profile of $T$. rubrum. For instance, $T$.
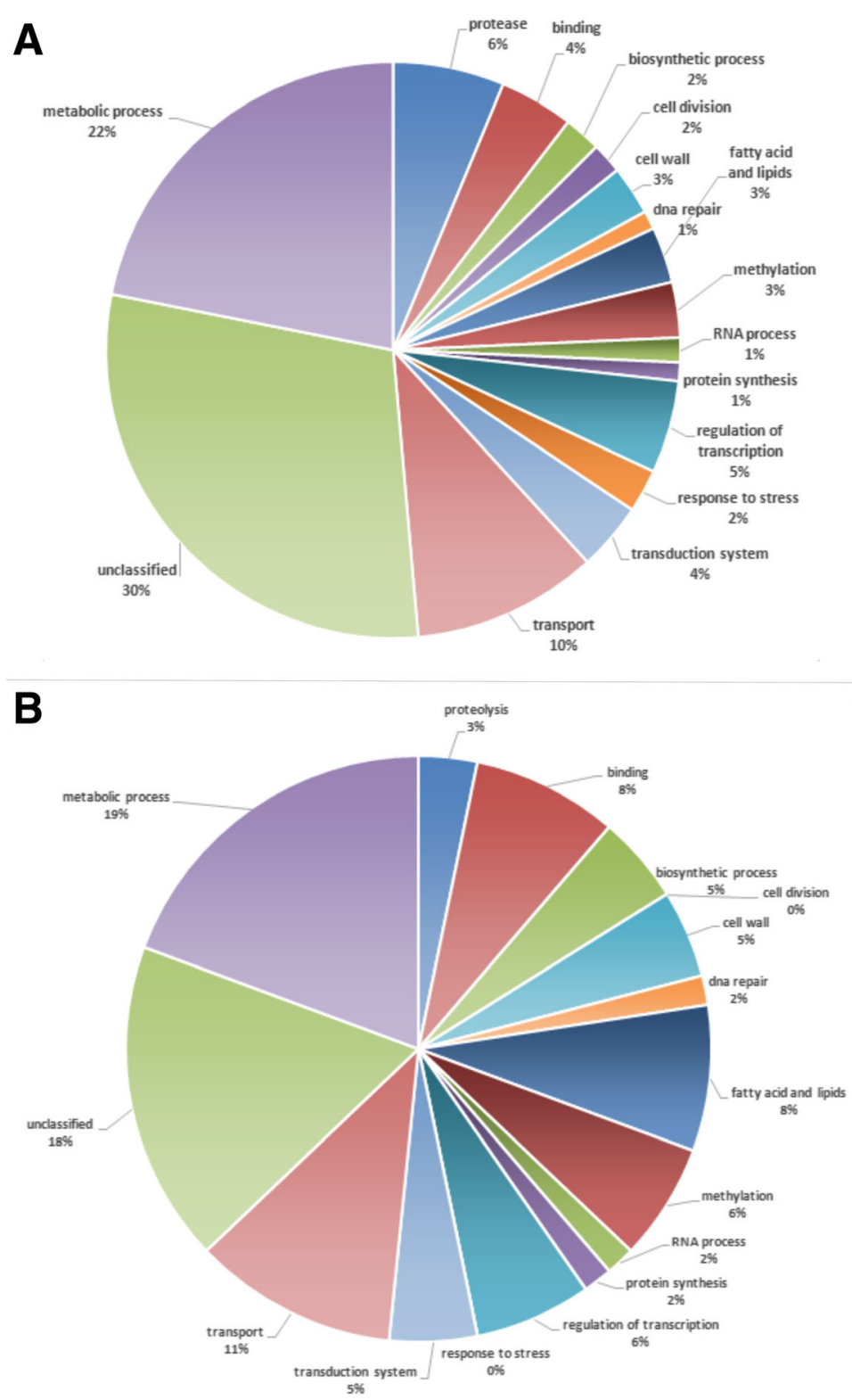

Fig. 2 Functional categorization of differentially expressed genes $(p<0.05)$ on MMK (a) and MME (b) 
rubrum grown on keratin showed enrichment for genes belonging to the proteolysis and stress response categories. On the other hand, enrichment for genes involved in fatty acid and lipid metabolism, transcription regulation process and cell wall components was observed when elastin was the protein source used (Fig. 2). Accordingly, 18 proteases and 7 lipases were differentially expressed in the two protein source conditions. This finding supports the involvement of protease secretion in keratin utilization in T. rubrum (Table 1).

\section{Functional categorization of T. rubrum genes involved in the response to trans-chalcone}

The gene expression profile of T. rubrum exposed to trans-chalcone was assessed to elucidate the mechanisms triggered by this chalcone. Genes above a cut off threshold of 5 and -5 in fold change in gene expression were submitted to Blast2GO, and then to a summarization using Revigo [12] algorithm. The most

Table 1 Protease- and lipase-encoding genes modulated during the growth of Trichophyton rubrum on keratin and elastin

\begin{tabular}{|c|c|c|c|}
\hline ID & Gene product name & Keratin & Elastin \\
\hline TERG_05923 & Metallopeptidase & -6.10 & - \\
\hline TERG_03293 & Hypothetical protein & -6.32 & - \\
\hline TERG_04809 & Metalloproteinase 2 & -8.12 & - \\
\hline TERG_05652 & Leucine aminopeptidase 1 & +20.71 & +8.05 \\
\hline TERG_04324 & Metalloproteinase 4 & +24.01 & - \\
\hline TERG_12606 & Secreted dipeptidyl peptidase & +5.03 & - \\
\hline TERG_03400 & Subtilisin-like protease 1 & +5.17 & - \\
\hline TERG_03104 & Signal peptidase i & +5.49 & - \\
\hline TERG_06552 & Aspartic-type endopeptidase & +5.82 & - \\
\hline TERG_04769 & Serine carboxypeptidase & +5.82 & - \\
\hline TERG_03248 & Metalloproteinase 3 & +6.72 & - \\
\hline TERG_02214 & Carboxypeptidase 2 & +6.79 & - \\
\hline TERG_08557 & Carboxypeptidase s1 & +7.24 & - \\
\hline TERG_08405 & Leucine aminopeptidase 2 & +8.31 & - \\
\hline TERG_05735 & Dipeptidyl peptidase 4 & +8.54 & - \\
\hline TERG_08201 & Subtilisin-like protease 5 & +9.06 & - \\
\hline TERG_03815 & Subtilisin-like protease 3 & +9.88 & - \\
\hline TERG_01617 & Subtilisin-like protease 4 & +9.98 & - \\
\hline TERG_01957 & Alkaline serine protease & - & +6.52 \\
\hline TERG_03459 & GDSL lipase acylhydrolase & - & +5.32 \\
\hline TERG_05317 & Lipase 1 & +11.54 & - \\
\hline TERG_04914 & Spo7-like protein & +5.40 & - \\
\hline TERG_00899 & Neutral ceramidase & +5.66 & - \\
\hline TERG_00127 & Secretory phospholipase a2 & +52.72 & +7.01 \\
\hline TERG_03747 & Phospholipase a2 & +6.79 & - \\
\hline
\end{tabular}

+: induction; -: repression representative differentially expressed categories based on GO functions are shown in Fig. 3. Overall, these genes are related to signal transduction, fatty acid and lipid metabolism, response to stress, pathogenesis, cell wall biosynthesis, and metabolic processes.

Noteworthy, trans-chalcone exposure down-regulated most genes involved in fatty acid synthesis (Table 2). In addition, genes involved in signal transduction like the cell wall integrity (CWI) pathway and Tor-mediated signaling were also modulated. These finding suggests a cross-talk between pathways to sense and respond to cellular stress caused by trans-chalcone (Table 2). Furthermore, alternative routes of energy metabolism were modulated after trans-chalcone exposure, such as beta-oxidation of fatty acids and glyoxylate cycle (Table 2).

\section{RT-qPCR and Western blot analysis}

The microarray results were validated by $\mathrm{qPCR}$ and the results demonstrated a strong positive correlation between the two techniques. Pearson correlation $r=0.91$ and $r=0.83$ for protein sources conditions and trans-chalcone exposure conditions, respectively (Fig. 4). The genes chosen for qPCR analysis were related to different biological processes: (i) proteases (metalloproteases, dipeptidyl protease, leucine aminopeptidase); (ii) lipases (phospholipase); (iii) cell wall biosynthetic pathway (beta glucosidase and chitin synthase); (iv) fatty acid and metabolic processes (fatty acid acetyl transferase, farnesyl transferase, acyl oxidase, copper transporter, glutamate kinase, and indoleamine dioxygenase).

In addition to the transcriptional pattern, we also assessed the expression of MAPK after T. rubrum exposure to trans-chalcone. The gene encoding CMGC MAPK in T. rubrum is homologous to MAPK 44/42 in Aspergillus fumigatus (score 729, e-value: 0.0, and 85\% identity). Here we evaluated the activation of CMGC MAPK by analyzing the phosphorylation levels of this MAP kinase after exposure to trans-chalcone. The results showed that MAPK was phosphorylated in response to trans-chalcone within the first hour of exposure, followed by a decrease thereafter (1 day). No phosphorylation was observed after 3 days (Fig. 5a). In addition, quantitative RT-PCR was carried out to correlate modulation of the gene encoding CMGC MAPK (TERG_00832) with protein expression (Fig. 5b). This analysis showed up-regulation of mapk transcription levels at $1 \mathrm{~h}$, followed by a decrease after 1 day of exposure and little changes in transcription levels after 3 days of exposure to trans-chalcone. Noteworthy, our microarray data mainly showed down-regulation of the CWI pathway at the time points analyzed and did not indicate any changes in the modulation of mapk gene transcription levels, 


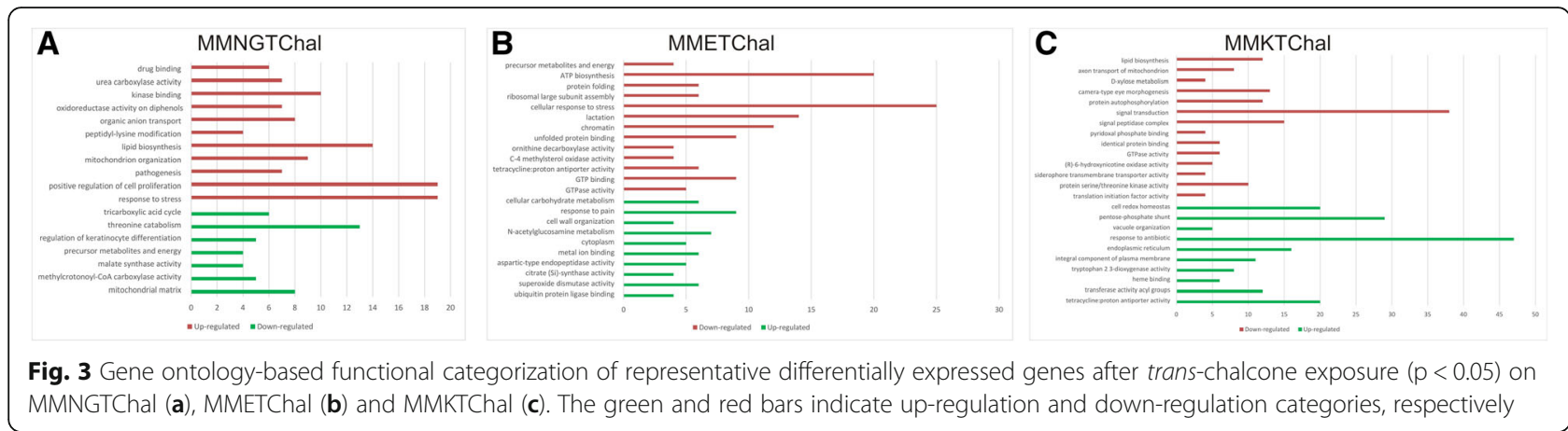

which probably is due to time points evaluated in our microarray data.

\section{Discussion}

The microarray data suggested slight differences in transcriptional profile of $T$. rubrum grown on different protein sources (keratin or elastin). The main differences were related to the modulation of proteases and some lipases in the keratin condition and to the modulation of genes belonging mainly to the lipase category in the elastin condition. Noteworthy, the low number in genes modulated for elastin substrate may be a result of T. rubrum lifestyle, since this fungus is more adapted to use keratin (a component of outermost layer of skin) than elastin (a substrate mainly found in dermis composition). Alike, we believe that the slight changes in transcript levels in MMETChal condition are due to the subtle changes in whole fungal metabolic machinery during T. rubrum grown on elastin substrate.

Four main categories can be highlighted in the transcriptional pattern of T. rubrum exposed to trans-chalcone: fatty acid and lipid metabolism, stress response, CWI pathway, and alternative energy metabolism.

\section{Trichophyton rubrum grown on keratin and elastin focus} on proteases, lipases, and heat shock proteins on focus Simulation of the host milieu is widely employed in investigations to assess putative virulence factors. Within this context, liquid media containing keratin powder, protein and even macerated skin have been used to elucidate key aspects of T. rubrum pathophysiology [6].

The establishment of dermatophyte infections is mainly attributed to their ability to adhere to host tissues and to adapt to this environment. Thereafter dermatophytes need to take up nutrients, which is mediated by the induction of specific enzymes such as lipases and proteases, particularly keratinases through sensing of skin $\mathrm{pH}$. Heat shock proteins may be involved in this process through a complex regulatory network in cooperation with PacC transcription factor [13]. Recent reports demonstrated that pacC, hsf1 (heat shock transcription factor), $c d c 37$ co-chaperone, and hsp 70 are markedly induced during the growth of dermatophytes on keratin sources at $37^{\circ} \mathrm{C}$ [3]. Additionally, in a previous study, the inhibition of Hsp90 decreased the ability of T. rubrum to grow on nail fragments [14]. Our microarray data showed that the growth of $T$. rubrum on a keratin source led to the up-regulation of hsp70-like (Terg_06505), hsp90-like (Terg_06963), and hsp88-like (TERG_07658).

Proteases are the most studied virulence factors of dermatophytes as they are required for nutrient uptake during the colonization of host tissue and the abundance of these proteins in the dermatophyte genome highlights their importance in the dermatophyte lifestyle $[5,15]$. Proteases are divided into endoproteases and exoproteases. Endoproteases include aspartic proteases, serine proteases (subtilisin family S8A) and metalloproteinases, while the exoprotease group comprises leucine aminopeptidases (Lap 1 and Lap2) and dipeptidyl-peptidases IV and V. Endo- and exoproteases act together in protein digestion $[16,17]$.

Our data showed that keratin promoted the induction of 15 genes encoding proteases, with expressive induction of the mep4 and lap1 genes (Table 1), also demonstrating that exo- and endoproteases are equally important for efficient keratin degradation. Furthermore, exposure to trans-chalcone promoted down-regulation of the genes encoding Mep 3, Sub 5, and signal peptidase, as well as of the genes encoding Hsp 70 like-protein, Hsp 88-like protein, and Hsp 90 like-protein (Additional file 1: Table S1).

In the elastin condition, two lipases and two proteases were found to be up-regulated, suggesting that lipases are as important as proteases during T. rubrum growth on elastin substrates (Table 1). In this sense, it worth to note that skin composition is also enriched with lipids, and the genomes of Arthroderma benhaminae and $T$. verrucosum contain 16 genes encoding lipases [4].

\section{Trans-chalcone exposure shift to the lipid routes by activating alternative energy metabolism}

Exposure of $T$. rubrum to trans-chalcone promoted changes in lipid and fatty acid metabolism. Genes 
Table 2 Main categories modulated in response to trans-chalcone* exposure

\begin{tabular}{|c|c|c|c|}
\hline ID & Gene product name & Fold change & Condition \\
\hline \multicolumn{4}{|c|}{ Transduction signaling } \\
\hline TERG_01124 & RAN-interacting protein & +5.37 & MMK + TChal \\
\hline TERG_05617 & Hypothetical protein & -5.29 & MMK + TChal \\
\hline TERG_05744 & GTP-binding protein & -6.87 & MMK + TChal \\
\hline TERG_02263 & Hypothetical protein & -5.29 & MMK + TChal \\
\hline TERG_04042 & Serine threonine protein kinase & -8.07 & MMK + TChal \\
\hline TERG_00315 & RAN protein kinase & -6.02 & MMK + TChal \\
\hline TERG_00077 & $\begin{array}{l}\text { Eukaryotic peptide chain release Factor GTP-binding } \\
\text { subunit }\end{array}$ & -6.72 & MMK + TChal \\
\hline TERG_04867 & SAM and $\mathrm{pH}$ domain-containing protein & -11.78 & MMK + TChal \\
\hline TERG_07136 & Farnesyltransferase beta subunit ram1 & $-9.29 /-5.37$ & $\begin{array}{l}\text { MMK + TChal/MMNG } \\
+ \text { TChal }\end{array}$ \\
\hline TERG_05617 & Hypothetical protein & -12.39 & MMNG+TChal \\
\hline TERG_00749 & Guanine nucleotide exchange & -15.78 & MMNG+TChal \\
\hline TERG_04523 & CMGC CDKL CRK7 protein kinase & -5.02 & MMNG+TChal \\
\hline TERG_01365 & GTP-binding protein & -5.2 & MMNG+TChal \\
\hline TERG_11963 & DEAD/DEAH box RNA helicase & -7.48 & MMNG+TChal \\
\hline TERG_01365 & GTP-binding protein & -5.2 & MMNG+TChal \\
\hline TERG_05987 & GTP-binding protein & +8.17 & MMNG+TChal \\
\hline TERG_01693 & Acyl oxidase & -6.42 & MMNG+TChal \\
\hline TERG_02422 & RHO GTPase activator & +2.18 & MME + TChal \\
\hline TERG_00689 & AUR protein kinase & +3.34 & MME + TChal \\
\hline TERG_07570 & G-protein signaling & -2.35 & MME + TChal \\
\hline TERG_04086 & GTP-binding protein 1 & -2.36 & MME + TChal \\
\hline TERG_00548 & Elongation factor 1 alpha & -2.6 & MME + TChal \\
\hline TERG_05987 & GTP-binding protein & -3.32 & MME + TChal \\
\hline \multicolumn{4}{|c|}{ Fatty acid and lipid metabolism } \\
\hline TERG_11538 & 3-oxoacyl-(acyl-carrier-protein) reductase & $\begin{array}{l}-7.45 / \\
-10.61\end{array}$ & MMK + TChal/MMNG_TChal \\
\hline TERG_11813 & FAD binding domain-containing protein & +5.95 & MMK + TChal \\
\hline TERG_11814 & FAD dependent protein & +10.63 & MMK + TChal \\
\hline TERG_08235 & Long-chain fatty alcohol oxidase & -5.52 & MMNG+TChal \\
\hline TERG_04851 & Acyl binding protein family & -2.30 & MMNG+TChal \\
\hline TERG_02787 & Fatty acid synthase S-acetyl transferase & -5.60 & MMNG+TChal \\
\hline DW707302.1 & Enoyl reductase & +4.09 & MME + TChal \\
\hline TERG_07644 & Ketoacyl reductase & +3.38 & MME + TChal \\
\hline \multicolumn{4}{|c|}{ TCA and glyoxylate cycle } \\
\hline TERG_03483 & Carnitine acetyltransferase & -5.48 & MMK + TChal \\
\hline DW687355.1 & Adenylsuccinate lyase & -15.01 & MMK + TChal \\
\hline TERG_05484 & Acyl dehydrogenase & +6.35 & MMK + TChal \\
\hline TERG_01281 & Malate glyoxomal & +5.11 & MMNG+TChal \\
\hline TERG_01052 & Succinyl ketoacyl transferase & +5.1 & MMNG+TChal \\
\hline DW700277.1 & Citrate synthase & -6.27 & MMNG+TChal \\
\hline TERG_05484 & Acyl dehydrogenase & +6.35 & MMK + TChal \\
\hline TERG_04250 & Carnitinyl- dehydratase & +2.13 & MME + TChal \\
\hline TERG_01271 & Isocitrate lyase & +2.04 & MME + TChal \\
\hline TERG_01272 & Methylcitrate mitochondrial & $+2.49 /+5.92$ & MME + TChal/MMNG+TChal \\
\hline
\end{tabular}

*TChal: trans-chalcone added at $0.24 \mu \mathrm{g} / \mathrm{mL}$. +: induction; -: repression 


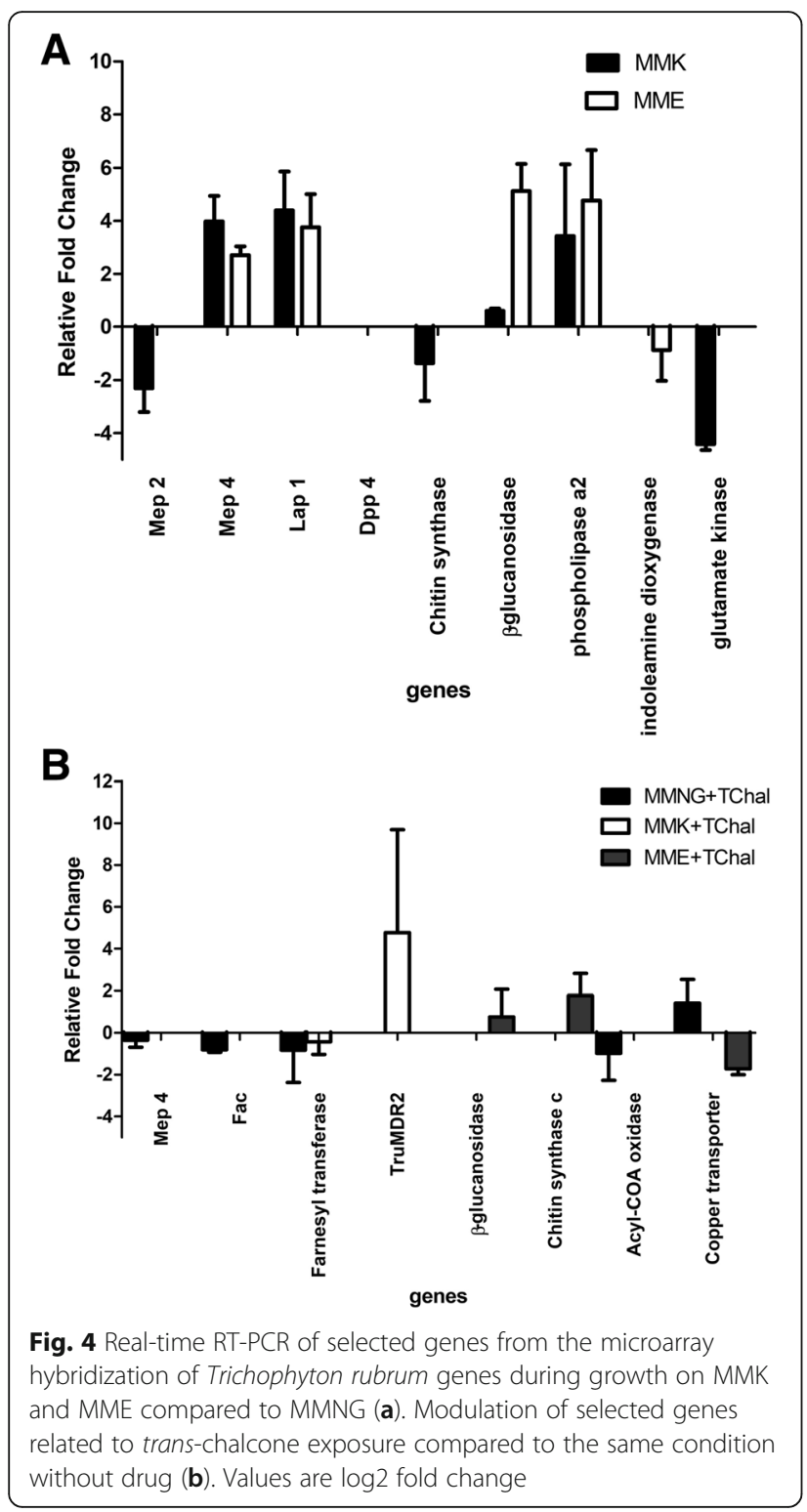

encoding enzymes involved in the early steps of fatty acid synthesis were generally down-regulated, whereas genes encoding enzymes belonging to the last steps of fatty acid elongation, such as enoyl-reductase and ketoacyl-reductase, were up-regulated (Table 2).

We suggest that impairment of the first steps of fatty acid synthesis led to an imbalance in the pathways involved in the supply of acetyl-CoA molecules. In this respect, $\beta$-oxidation is activated in order to supply acetyl-CoA molecules through enzymatic reactions catalyzed by acyl dehydrogenase and ketoacyl-thiolases. The resulting acetyl-CoA can enter the mitochondrial tricarboxylic acid (TCA) cycle [18]. Moreover, citrate from the TCA cycle can be converted to isocitrate, which becomes a glyoxylate cycle substrate, and the resulting malate may enter gluconeogenesis [19]. Consistently, our data showed up-regulation of the genes encoding acyl dehydratases (mediator of first committed steps in fatty acid $\beta$ oxidation) and ketoacyl thiolases. In addition, exposure to trans-chalcone changed the expression of genes related to the glyoxylate and TCA cycles, with up-regulation of the genes encoding isocitrate lyase, succinate lyase, and succinyl-ketoacyl transferase (Table 2).

Moreover a previous work has shown that trans-chalcone activity against dermatophytes relies on the down-regulation of fatty acid synthesis $\beta$ subunit (fas 1 ), a decrease in ergosterol content, and moderate inhibition of FAS enzymatic activity [8]. It is noteworthy to mention that palmitate synthesis (the final product of the FAS multienzyme complex [20]) needs the input of acetyl-CoA molecules, reducing agents (NADPH), and energy from ATP. So, its proper synthesis requires the coordinated use of multiple energy metabolic pathways like the TCA and glyoxylate cycles [21]. Supporting this idea, we also demonstrated changes in the modulation of genes involved in acetyl-CoA transport, such as carnitine and citrate synthase.

Taken together, the overall effects promoted by trans-chalcone exposure may be related to impairment in fatty acid synthesis and concomitant changes in energy metabolic pathways involved in acetyl-CoA supply. Finally, lipid burning seems to occur as evidenced by changes in the $\beta$-oxidation of fatty acids, which confers to trans-chalcone a thermogenic feature that might be related to the reduction in ergosterol content and consequent cell wall damage previously described by [8].

\section{Cross-talk events in the cell wall integrity pathway are activated by trans-chalcone}

The cell wall is a dynamic structure that is essential to maintain cell shape and to protect against environmental threats. In this regard, the cell wall is remodeled according to developmental stage or after drug exposure. The rearrangements in cell wall composition ensure the structural integrity during conditions that compromise cell wall and/or membrane integrity [22].

The CWI-signaling pathway is activated to protect the cell wall against threats or even during cell growth. The stimuli are sensed by cell wall mechanosensors located on the plasma membrane, such as Mid2 and Mtl1, and by Wsc protein family members. The signals are then transmitted to small Rho1 GTPase, which is activated by regulatory inputs from guanine nucleotide exchange factors (GEFs), Rom1 and Rom2. Next, Rho1p activates protein kinase $\mathrm{C}(\mathrm{Pkc1})$. PKC is located upstream in the MAPK (mitogen-activated protein kinase) signaling cascade. Finally, effectors of Rho1 are $\beta$-1,3-glucan synthase and $\beta-1,6$-glucan synthase, proteins of the actin cytoskeleton and secretory vesicles [22]. 


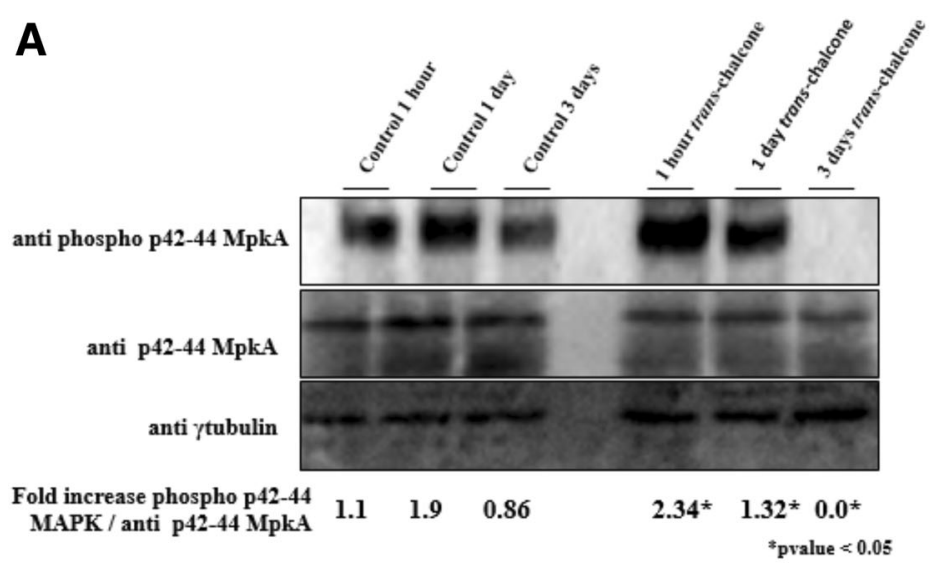

B

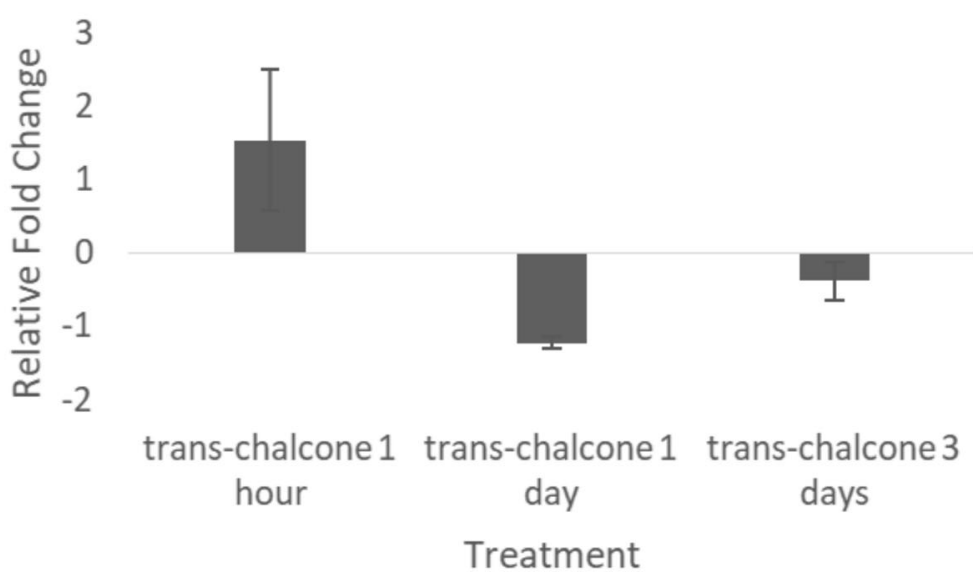

Fig. 5 Western blot results of Mapk expression and Mapk phosphorylation. Trans-chalcone was added or not (control) to the medium for $1 \mathrm{~h}, 1$ day, and 3 days. Antiphospho-p44/42MAPK antibody was used to detect MpkA phosphorylation. The $\gamma$-tubulin antibody was used as control (a). Gene expression analysis of CMGC/MAPK Erk1 in T. rubrum by qPCR. The fungus was grown in medium with trans-chalcone $(0.24 \mu \mathrm{g} / \mathrm{mL}) \mathrm{or}$ without the compound (control) for $1 \mathrm{~h}, 1$ day, and 3 days (b)

During stress, the cell wall undergoes remodeling through a reinforce by increase of chitin amounts and incorporation of certain cell wall proteins. This rearrangement occurs through cross-talk between different signaling pathways $[23,24]$. Additionally, any changes in the polarized growth of filamentous fungi are related to impairment of cell wall formation as well as to conditions that indirectly affect cell wall biosynthesis. Thus, to enhance the signaling capabilities of the CWI pathway in order to deal with diverse stress conditions, this pathway cross talks with other signaling pathways or proteins [25]. The interconnection between these responses signaling to pathways of cell wall assembly needs to be widely understood. Within this context, a previous study that compared the response of $A$. niger to caspofungin (inhibitor of $\beta-1,3$ glucan synthase) [26] and fenpropimorph (Saccharomyces cerevisiae inhibitor of erg2 and erg 24 in ergosterol biosynthesis) [27] using the microarray technique showed common responses, promoting changes in signaling pathways such as CWI signaling and Tor signaling and in genes involved in cell membrane composition [24].

In agreement with previous studies, our microarray data showed that exposure of T. rubrum to trans-chalcone promoted changes in three distinct signaling pathways, suggesting considerable interaction between the Tor signaling, CDK kinase, and MAPK pathways. In addition, Western blot analysis revealed the activation of MAPK after exposure to trans-chalcone for $1 \mathrm{~h}$.

\section{Conclusions}

In conclusion, the present results suggest that the mode of action of trans-chalcone is related to pronounced changes in fungal metabolism, promoting a shift to lipid metabolism and activating a cross-talk between signaling pathways related to CWI. In addition, we showed that trans-chalcone acts on virulence factors such as proteases as well as modulates heat shock proteins. 
Notwithstanding,we also aware that these interconnections need to be further investigated with association of protein and gene expression profiles.

\section{Methods}

Trichophyton rubrum strain and growth conditions

Trichophyton rubrum CBS 118892 was cultured on Sabouraud dextrose agar (Oxoid, Hampshire, England) at $28^{\circ} \mathrm{C}$, as described previously [28]. Conidial suspensions were obtained from 15 -day-old plates. The conidial concentration was determined in a Neubauer chamber and approximately $1.6 \times 10^{6}$ conidia were added to 20 $\mathrm{mL}$ of liquid Sabouraud and incubated for $72 \mathrm{~h}$ at $28{ }^{\circ} \mathrm{C}$ under shaking at $150 \mathrm{rpm}$. The resulting mycelia were incubated under six different conditions: i) control medium (MMNG): Cove's minimal medium [29] containing $70 \mathrm{mM}$ nitrate (Sigma Aldrich, St. Louis, MO, USA) and $50 \mathrm{mM}$ glucose (Sigma Aldrich); ii) keratin medium (MMK): Cove's minimal medium supplemented with $0.5 \%$ bovine keratin; iii) elastin medium (MME): Cove's medium supplemented with $0.25 \%$ elastin (Sigma Aldrich); iv) MMNG+TChal: MMNG medium containing $0.24 \mu \mathrm{g} / \mathrm{mL}$ of trans-chalcone (Sigma Aldrich); v) MMK + TChal: MMK containing $0.24 \mu \mathrm{g} / \mathrm{mL}$ of trans-chalcone, and vi) MME + TChal: MME containing $0.24 \mu \mathrm{g} / \mathrm{mL}$ of trans-chalcone. The $\mathrm{pH}$ of the medium was 5.0 in all conditions and the cultures were incubated for 3,7 , and 14 days at $28^{\circ} \mathrm{C}$ under shaking $(130 \mathrm{rpm})$. The concentration of trans-chalcone was based on its minimal inhibitory concentration as reported previously [9].

\section{Total RNA extraction}

Total RNA was extracted using the Illustra RNAspin Isolation Kit (GE Healthcare, Little Chalfont, Buckinghamshire, UK) following manufacturer's instructions. The quality and concentration of the RNA were checked by measuring the OD 260/280 and OD 260/230. RNA degradation was analyzed by microfluidic electrophoresis using Agilent 6000 RNA Nano chips in an Agilent 2100 Bioanalyzer (Agilent Technologies, Santa Clara, CA, USA). Samples with an RNA integrity number (RIN) $\geq$ 9.0 were used.

\section{Microarray hybridization}

The gene expression profile of T. rubrum grown in each of the six conditions was analyzed using the custom slides of the Agilent $4 \times 44 \mathrm{~K}$ High Density Oligonucleotide Array, as previously described [10]. About thirty-three nanograms of RNA from each incubation time (3, 7 and 14 days) were pooled for each condition. These pooled RNA from each condition was used as a template to generate double strands of cDNA and cRNA labeled with cyanine (Cy3)-CTP using the Agilent Low
Input Amplification Kit (Agilent Technologies, Santa Clara, CA, USA). Complementary RNA hybridization was performed using Agilent's SureHyb chambers in a rotator oven for $18 \mathrm{~h}$ at $60{ }^{\circ} \mathrm{C}$. Two biological replicates were used for each condition. In addition to the functional genes of T. rubrum, internal control probes were included on the custom slides. The wash steps of the manufacturer's protocol were followed.

\section{Analysis of microarray data}

The oligo-mRNA array slides were scanned with a DNA microarray scanner (Agilent Technologies) and the Agilent Feature Extraction 10.5 software [30] was used to extract the hybridization signals. The analysis was performed by pairwise comparison using a moderated t-test algorithm as follows: MMK + TChal x MMK; MME + TChal x MME, MMNG+TChal x MMNG, and MMK + TChal $x$ MME + TChal. The quantitative microarray data were normalized by quantile normalization and were analyzed using the Gene Spring GX 12.6 Bioinformatics Platform (http://www.agilent.com/chem/genespring) according to manufacturer's instructions. Statistical analysis was performed by ANOVA $(P<0.05)$ using a fold change cut-off $\geq 5.0$ for most conditions, except for MME+ TChal in which a fold change $\geq 2$ was used as cut-off. The Benjamini-Hochberg algorithm was used to calculate the false discovery rate, except for comparison between MME + TChal versus MME, in which Storey's bootstrapping approach was applied. The different statistical parameters employed for MME + TChal versus MME is due to the fewer changes in transcript levels for this comparison. Each EST with its corresponding protein-coding gene in the T. rubrum genome was mapped using Blastx (e-value 1e-5). In addition, putative annotations were retrieved using Blastx and the biological function of mRNA was assessed through GO terms obtained with BayGO [11]. The genes without any associated GO term were called "unclassified". The raw data are deposited in the Gene Expression Omnibus (GEO) (www.ncbi.nlm.nih.gov/geo) database under accession number: GSE123979.

\section{RT-qPCR}

The microarray expression data were validated by RT-qPCR using a set of 12 genes (Table 3). The oligonucleotide sequences were retrieved from IDT DNA "primer quest" tool (http://www.idtdna.com/primerquest/Home/Index) The selected genes belongs to main categories modulated by trans-chalcone exposure or during T. rubrum growth on protein sources. Complementary DNA was synthesized from $1000 \mathrm{ng}$ of total RNA in a $20-\mu \mathrm{L}$ reaction volume using the RevertAID H Minus First Strand cDNA Synthesis Kit (Fermentas ${ }^{\oplus}$ ) according to manufacturer's instruction. The quantitative RT-PCR experiments were performed in triplicate 
Table 3 Set of primers used in the qPCR assays

\begin{tabular}{|c|c|c|c|}
\hline ID & Gene product name & Sequence $5^{\prime}-3^{\prime}$ & $\mathrm{bp}$ \\
\hline TERG_11895 & Fatty acid acetyl transferase (Fac) & $\begin{array}{l}\text { Fwd: 5'-ATGCGCCATGTTCTGTCTCA-3' } \\
\text { Rev.: 5'- TGGTGAAGCGAACAACGAGA-3' }\end{array}$ & 133 \\
\hline TERG_04809 & Extracellular metalloproteinase (Mep 2) & $\begin{array}{l}\text { Fwd: 5'- GGCACAAGACCAAGAGACCC-3' } \\
\text { Rev.: 5'- AGGCTTGTTGTCCGAGTCAG -3 }\end{array}$ & 145 \\
\hline TERG_06242 & Beta-glucan glucosidase & $\begin{array}{l}\text { Fwd: 5'- CTCAATGTAGCGGCGGGTAT-3' } \\
\text { Rev:: 5'- CACAAAGACTCGGACCCCAA-3' }\end{array}$ & 114 \\
\hline TERG_05652 & Aminopeptidase leucine (LAP1) & $\begin{array}{l}\text { Fwd: 5'- TCCAGGCTGCCATCAATAC-3' } \\
\text { Rev:: 5'- GAATAGTGGCAATGATGCTGTG-3' }\end{array}$ & 99 \\
\hline TERG_02562 & Chitin synthase $c$ & $\begin{array}{l}\text { Fwd: 5'-TTGCCGGTCTAGGTGTTTAC-3' } \\
\text { Rev:: 5'-CATGCCTATCTGGGTGGTATATT-3' }\end{array}$ & 101 \\
\hline TERG_00694 & Glutamate kinase & $\begin{array}{l}\text { Fwd: 5'-ATCCTGATGCTCGGGTTATTG-3' } \\
\text { Rev.: 5'-CCACTATCTTTGAGCCCATACC-3' }\end{array}$ & 111 \\
\hline TERG_04324 & Extracellular metalloproteinase (Mep4) & $\begin{array}{l}\text { Fwd: 5'-GCATGGACTTATGCTTGCGG-3' } \\
\text { Rev.: 5'-TGGATATCTGGGGAAGGCGA }\end{array}$ & 131 \\
\hline TERG_07136 & Farnesyl transferase & $\begin{array}{l}\text { F:5'-AGGCGTTACCTTGATCGATAG-3' } \\
\text { R:5'-GCCATCTCCAACTACACCATTA-3' }\end{array}$ & 91 \\
\hline TERG_01329 & Cooper transporter & $\begin{array}{l}\text { F: 5'- CTCACGGCCAAAGCTATCA-3' } \\
\text { R:5'- TGATCCAGGCGGTGATATTG-3' }\end{array}$ & 105 \\
\hline TERG_02909 & Acyl oxidase & $\begin{array}{l}\text { F:5'- TGAGAGAGGCCAGTCCAATA-3' } \\
\text { R:5'- TGCTGAATGAGGGAAAGGATAC-3' }\end{array}$ & 102 \\
\hline TERG_00127 & Phospholipase a2 & $\begin{array}{l}\text { F: 5'- GCCACGAGGATACGACTTTAT-3' } \\
\text { R:5'- ATCAACCTTCTTGCGGTAGTC-3' }\end{array}$ & 106 \\
\hline TERG_02134 & Indoleamine-dioxygenase & $\begin{array}{l}\text { F:5'- CTGCAGCGTATGCCAATAAAG-3' } \\
\text { R:5'- GAGCAGTGAGATCAGGTAACTC-3' }\end{array}$ & 103 \\
\hline TERG_08613 & TruMDR2 & $\begin{array}{l}\text { F: } 5^{\prime}-\text { GCACTGATCTGCAGCTCGACC-3' } \\
\text { R:5' CCAACGTCATCCTCCCAGAC-3' }\end{array}$ & 91 \\
\hline TERG_00832 & CMGC/MAPK protein kinase (Erk1) & $\begin{array}{l}\text { F: 5'- CTTGAAGCCCGGTAACCTATT-3' } \\
\text { R: 5'- CGGTCATATATCCAGCGTTCTC -3' }\end{array}$ & 113 \\
\hline TERG_07904 & *Beta-tubulin & $\begin{array}{l}\text { F: 5'- AACATGATGGCTGCCACTGA-3' } \\
\text { R: 5' - AAGATGGCAGAGCAGGTAAGGT-3' }\end{array}$ & 253 \\
\hline
\end{tabular}

*Beta tubulin was described by [32]

using the SYBR Taq Ready Mix Kit (Sigma) on an Mx3300 QPCR system (Stratagene), as previously described [8]. The cycling conditions included an initial PCR step at $94{ }^{\circ} \mathrm{C}$ for $10 \mathrm{~min}$, followed by 40 cycles of $94{ }^{\circ} \mathrm{C}$ for $2 \mathrm{~min}, 60^{\circ} \mathrm{C}$ for $1 \mathrm{~min}$, and $72^{\circ} \mathrm{C}$ for $1 \mathrm{~min}$. At the end of each PCR cycle, a dissociation curve was constructed. Expression levels were calculated by the comparative $2^{-\Delta \Delta C t}$ method [31] using beta-tubulin as normalizer. The reference for validation of the microarray data was Cove's minimal medium for keratin or elastin comparison and the respective condition without trans-chalcone for comparison with the drug-containing medium the cultures for 3,7 , and 14 days at $28^{\circ} \mathrm{C}$ under shaking $(130 \mathrm{rpm})$. The results are reported as the mean \pm standard deviation of three independent experiments.

\section{Protein extraction and Western blot analysis of phosphorylated MpkA}

In order to assess the phosphorylated status of MpkA, a T. rubrum conidial suspension was obtained from 15-day-old Sabouraud agar plates. Approximately $1 \times 10^{7}$ conidia were added to $20 \mathrm{~mL}$ of liquid Sabouraud under shaken $(130 \mathrm{rpm})$ for $72 \mathrm{~h}$ at $28^{\circ} \mathrm{C}$. The mycelia were transferred to minimal medium (MMNG) supplemented with $0.24 \mu \mathrm{g} / \mathrm{mL}$ of trans-chalcone and incubated for 1 h, 1 day and 3 days at $28^{\circ} \mathrm{C}$ under shaking. The control was left untreated. After incubation, the mycelia were retrieved and frozen at $-80^{\circ} \mathrm{C}$ until use. Prior to use, the mycelia were lyophilized. Total protein was extracted and the mycelia were ground in liquid nitrogen with a mortal and a pestle. About $0.5 \mathrm{~mL}$ of lysis buffer described in reference [33] was added to the ground mycelium and submitted to vigorous agitation. The lysis buffer contained $10 \%(\mathrm{v} / \mathrm{v})$ glycerol, $50 \mathrm{mM}$ Tris- $\mathrm{HCl}$, $\mathrm{pH} 7.5,1 \%(\mathrm{v} / \mathrm{v})$ Triton X-100, $150 \mathrm{mM} \mathrm{NaCl}, 0.1 \%(\mathrm{w} / \mathrm{v})$ SDS, $5 \mathrm{mM}$ EDTA, $50 \mathrm{mM} \mathrm{NaF}, 5 \mathrm{mM}$ sodium pyrophosphate, $50 \mathrm{mM} \beta$-glycerophosphate, $5 \mathrm{mM}$ sodium orthovanadate, $1 \mathrm{mM}$ PMSF, and $1 \mathrm{X}$ Complete Mini Protease Inhibitor (Roche Applied Science). The samples were kept on ice. The extracts were then centrifuged at $20,000 \mathrm{~g}$ for $1 \mathrm{~h}$ at $4{ }^{\circ} \mathrm{C}$. The supernatants were collected 
and kept at $-80{ }^{\circ} \mathrm{C}$ until use. The total protein concentration was determined by the Hartree method [34]. About $50 \mu \mathrm{g}$ of protein obtained from each condition was resolved on 12\% (w/v) SDS-PAGE [35] and transferred to polyvinylidene difluoride (PVDF) membranes (BioRad) using submerged method according to manufacturer's instructions.

MAP kinase phosphorylation was examined using anti-phospho p44/42 (9101; Cell Signaling Technologies) and anti-p44/42 (9102; Cell Signaling Technologies) antibodies diluted 1:1000 in TBST buffer containing 5\% BSA for $16 \mathrm{~h}$ at $4{ }^{\circ} \mathrm{C}$, according to manufacturer's instructions. The primary antibody was detected with HRP-conjugated secondary antibody raised in rabbit (Thermo Scientific) by incubation for $2 \mathrm{~h}$ at room temperature. The $\gamma$-tubulin antibody was used as the control of the assay. Chemiluminescent detection was conducted using the ECL Prime Western Blot Detection Reagent (GE HealthCare). The images were generated by exposing the membrane to the ChemiDoc XRS gel imaging system (BioRad). The ImageJ software was used for densiometric analysis.

\section{Additional file}

Additional file 1: Table S1. Genes modulated by Trichophyton rubrum during growth on protein sources, and after trans-chalcone exposure. (DOC $675 \mathrm{~kb})$

\section{Abbreviations}

CWI: Cell wall Integrity; fas: Fatty acid synthase; GEFs: Guanine nucleotide exchange factors; GEO: Gene Expression Omnibus; GO: Gene ontology; MAPK: Mitogen Activated Protein Kinase; MME: Elastin medium; MMETChal: MME containing trans-chalcone; MMK: Keratin medium; MMKTChal: MMK containing trans-chalcone; MMNG: Cove's minimal medium containing nitrate and glucose; MMNG+TChal: MMNG medium containing trans-chalcone; PKC: protein kinase C; RT-PCR: Reverse Transcription PCR; TCA: Tricarboxylic acid

\section{Acknowledgements}

We thank the staff of the Biotechnology Unit, UNAERP, for their general support and Professor Nilce M. Martinez-Rossi for kindly providing the $T$. rubrum strain CBS.

\section{Funding}

This work was supported by grants from the São Paulo Research Foundation - FAPESP [Grant Nos. 2016/22701-9 and 2012/02920-7 and 2015/23435-8 to T.A.B. and 2016/16881-4 to I.S.M]. The funding agency had no role in the design, collection, analysis, or interpretation of the data or in writing the manuscript.

\section{Availability of data and materials}

The datasets generated and/or analysed during the current study are available in the Gene Expression Omnibus (GEO) database at http://www. ncbi.nlm.nih.gov/geo under accession number GSE123979.

\section{Authors' contributions}

AL and MM conceived the study. TAB designed the study, performed the bioinformatics analysis, and laboratory experiments. MEF. and PRS performed the bioinformatics analysis. CM hybridized the microarrays. GAP contributed with the hybridization experiments and microarray platform. MCR performed the Western blot assay. ISM and BAMC performed the microbiology assays.
IM customized the T. rubrum microarray slides and revised the manuscript. $\mathrm{TAB}, \mathrm{ROB}$., and AL. wrote the manuscript. All authors have read and approved the manuscript.

Ethics approval and consent to participate

Not applicable.

\section{Consent for publication}

Not applicable.

\section{Competing interests}

The authors declare that they have no competing interests.

\section{Publisher's Note}

Springer Nature remains neutral with regard to jurisdictional claims in published maps and institutional affiliations.

\section{Author details}

${ }^{1}$ Unidade de Biotecnologia, Universidade de Ribeirão Preto, Av: Costábile Romano 2201, Ribeirão Preto, SP 14096-900, Brazil. ²Departamento de Genética, Faculdade de Medicina de Ribeirão Preto, Universidade de São Paulo, Ribeirão Preto, SP, Brazil. ${ }^{3}$ Departamento de Genética e Evolução, Centro de Ciências Biológicas e da Saúde (CCBS), Universidade Federal de São Carlos, São Carlos, Brazil. ${ }^{4}$ Instituto Federal do Sul de Minas - Campus Machado, Machado, Brazil.

Received: 21 December 2018 Accepted: 10 May 2019

Published online: 22 May 2019

\section{References}

1. Ameen M. Epidemiology of superficial fungal infections. Clin Dermatol. 2010;28(2):197-201.

2. Nenoff, P., et al., Mycology - an update. Part 1: dermatomycoses: causative agents, epidemiology and pathogenesis. J Dtsch Dermatol Ges, 2014. 12(3): p. 188-209; quiz 210, 188-211; quiz 212.

3. Martinez-Rossi NM, et al. Heat shock proteins in dermatophytes: current advances and perspectives. Curr Genomics. 2016;17(2):99-111.

4. Burmester, A., et al., Comparative and functional genomics provide insights into the pathogenicity of dermatophytic fungi. Genome Biol, 2011. 12(1): p. R7.

5. Martinez DA, et al. Comparative genome analysis of Trichophyton rubrum and related dermatophytes reveals candidate genes involved in infection. MBio. 2012;3(5).

6. Peres NT, et al. In vitro and ex vivo infection models help assess the molecular aspects of the interaction of Trichophyton rubrum with the host milieu. Med Mycol. 2016;54(4):420-7.

7. Martinez-Rossi NM, Peres NTA, Rossi A. Antifungal resistance mechanisms in dermatophytes. Mycopathologia. 2008;166:369-83.

8. Bitencourt TA, et al. Trans-chalcone and quercetin down-regulate fatty acid synthase gene expression and reduce ergosterol content in the human pathogenic dermatophyte Trichophyton rubrum. BMC Complement Altern Med. 2013:13:229.

9. Komoto $\Pi$, et al. Gene expression response of Trichophyton rubrum during coculture on keratinocytes exposed to antifungal agents. Evid Based Complement Alternat Med. 2015;2015:180535.

10. Bitencourt TA, et al. Transcription profile of Trichophyton rubrum conidia grown on keratin reveals the induction of an adhesin-like protein gene with a tandem repeat pattern. BMC Genomics. 2016:17:249.

11. Vencio RZ, et al. BayGO: Bayesian analysis of ontology term enrichment in microarray data. BMC Bioinformatics. 2006;7:86.

12. Supek F, et al. REVIGO summarizes and visualizes long lists of gene ontology terms. PLoS One. 2011;6(7):e21800.

13. Martinez-Rossi, N.M., et al., Heat shock proteins in dermatophytes: current advances and perspectives. Current Genomics, 2016. 17 p. DOl: https://doi. org/10.2174/1389202917666151116212437.

14. Jacob TR, et al. Heat shock protein 90 (Hsp90) as a molecular target for the development of novel drugs against the dermatophyte Trichophyton rubrum. Front Microbiol. 2015;6:1241.

15. Grumbt M, Monod M, Staib P. Genetic advances in dermatophytes. FEMS Microbiol Lett. 2011;320(2):79-86. 
16. Monod M, et al. Secreted proteases from pathogenic fungi. Int J Med Microbiol. 2002;292(5-6):405-19.

17. Zaugg C, et al. Trichophyton rubrum secreted and membrane-associated carboxypeptidases. Int J Med Microbiol. 2008;298(7-8):669-82.

18. Poirier $Y$, et al. Peroxisomal beta-oxidation--a metabolic pathway with multiple functions. Biochim Biophys Acta. 2006;1763(12):1413-26.

19. Strijbis K, Distel B. Intracellular acetyl unit transport in fungal carbon metabolism. Eukaryot Cell. 2010;9(12):1809-15.

20. Jenni $\mathrm{S}$, et al. Architecture of a fungal fatty acid synthase at 5 a resolution. Science. 2006;311(5765):1263-7.

21. Berg, J.T., JL; Stryer, L., Fatty acids are Synthesized and Degraded by Different Pathways. , In Biochemistry, W.H. freeman, Editor. 2002: New York.

22. Levin DE. Cell wall integrity signaling in Saccharomyces cerevisiae. Microbiol Mol Biol Rev. 2005;69(2):262-91.

23. Lagorce A, et al. Genome-wide analysis of the response to cell wall mutations in the yeast Saccharomyces cerevisiae. J Biol Chem. 2003;278(22): 20345-57.

24. Meyer $V$, et al. Survival in the presence of antifungals: genome-wide expression profiling of Aspergillus Niger in response to sublethal concentrations of caspofungin and fenpropimorph. J Biol Chem. 2007; 282(45):32935-48.

25. Fuchs BB, Mylonakis E. Our paths might cross: the role of the fungal Cell Wall integrity pathway in stress response and cross talk with other stress response pathways. Eukaryot Cell. 2009:8(11):1616-25.

26. Franzot SP, Casadevall A. Pneumocandin L-743,872 enhances the activities of amphotericin B and fluconazole against Cryptococcus neoformans in vitro. Antimicrob Agents Chemother. 1997;41(2):331-6.

27. Marcireau C, Guilloton M, Karst F. In vivo effects of fenpropimorph on the yeast Saccharomyces cerevisiae and determination of the molecular basis of the antifungal property. Antimicrob Agents Chemother. 1990;34(6):989-93.

28. Fachin AL, Maffei CML, Martinez-Rossi NM. In vitro susceptibility of Trichophyton rubrum isolates to griseofulvin and tioconazole. Induction and isolation of a resistant mutant to both antimycotic drugs. Mycopathologia. 1996;135(3):141-3.

29. Cove DJ. The induction and repression of nitrate reductase in the fungus Aspergillus nidulans. Biochim Biophys Acta. 1966;113(1):51-6.

30. Tan CS, et al. A review of feature extraction software for microarray gene expression data. Biomed Res Int. 2014;2014:p. 213656.

31. Schefe JH, et al. Quantitative real-time RT-PCR data analysis: current concepts and the novel "gene expression's CT difference" formula. J Mol Med (Berl). 2006;84(11):901-10.

32. Jacob TR, Peres NT, Persinoti GF, Silva LG, Mazucato M, Rossi A, MartinezRossi NM: rpb2 is a reliable reference gene for quantitative gene expression analysis in the dermatophyte Trichophyton rubrum. Med Mycol. 2012;50(4): 368-377.

33. Rocha MC, et al. The Aspergillus fumigatus pkcA G579R mutant is defective in the activation of the Cell Wall integrity pathway but is dispensable for virulence in a neutropenic mouse infection model. PLoS One. 2015;10(8): e0135195.

34. Hartree EF. Determination of Protein - Modification of Lowry Method That Gives a Linear Photometric Response. Analytical Biochemistry. 1972;48(2): 422.

35. Gallagher SR. One-dimensional SDS gel electrophoresis of proteins. Cur Protoc Immunol. 2006; Chapter 8: p. Unit 84.

Ready to submit your research? Choose BMC and benefit from:

- fast, convenient online submission

- thorough peer review by experienced researchers in your field

- rapid publication on acceptance

- support for research data, including large and complex data types

- gold Open Access which fosters wider collaboration and increased citations

- maximum visibility for your research: over $100 \mathrm{M}$ website views per year

At $\mathrm{BMC}$, research is always in progress.

Learn more biomedcentral.com/submissions 